\title{
The Application of Biophysical Techniques to Study Antimicrobial Peptides
}

\author{
Inês M. Torcato, ${ }^{1}$ Miguel A. R. B. Castanho, ${ }^{1}$ and Sónia T. Henriques ${ }^{1,2}$ \\ ${ }^{1}$ Instituto de Medicina Molecular, Faculdade de Medicina da Universidade de Lisboa, \\ 1649-028 Lisboa, Portugal \\ ${ }^{2}$ Institute for Molecular Bioscience, The University of Queensland, Brisbane, QLD 4072, Australia
}

Correspondence should be addressed to Sónia T. Henriques, s.henriques@imb.uq.edu.au

Copyright (C) 2012 Inês M. Torcato et al. This is an open access article distributed under the Creative Commons Attribution License, which permits unrestricted use, distribution, and reproduction in any medium, provided the original work is properly cited.

\begin{abstract}
The increasing bacteria resistance to conventional antibiotics has led to the need for alternative therapies. Being part of the human innate defence system and with a broad spectrum of activity against bacteria, viruses, protozoa, and cancer cells, antimicrobial peptides (AMPs) are a very promising alternative. The mechanism of action of AMPs seems to broadly correlate with their ability to target the bacterial cell membrane. To understand and improve their effect, it is of major importance to unravel their mechanism of action and, in particular, to understand the peptide-membrane binding. Several biophysical techniques such as fluorescence spectroscopy, circular dichroism, zeta potential determination, and atomic force microscopy can be used to achieve this goal. Characteristics of AMPs-membranes interactions and the use of these biophysical techniques will be discussed.
\end{abstract}

Keywords: Antimicrobial peptides, bacteria, model membranes, biophysical techniques

\section{Antimicrobial Peptides (AMPs)}

The effectiveness of antibiotics has become limited due to an increase in bacterial resistance; thus, the development of new antimicrobial agents that increase the effectiveness and reduce the side effects, in comparison to traditional treatments, is of major relevance. With a broad action spectrum, and less susceptiblilty to the development of bacterial resistance, AMPs have been regarded as an exciting alternative to classical antibiotics [1]. They exist in nearly all organisms as part of their innate immune system and have activity against bacteria, viruses, protozoa, and cancer cells $[2,3]$. By selecting the bacterial cell membrane over the host cell, AMPs induce bacterial cell lysis and death without being toxic to the host cells.

Generally, AMPs are short (less than 50 aminoacid residues), cationic, and have an amphipathic character [4]. Nevertheless, these peptides exhibit great sequence variability and can be divided in four major classes classified based on their secondary structure: $\alpha$-helical, $\beta$-sheet, looped, and extended [3-5]. AMPs with $\alpha$-helical structure commonly exist as unstructured monomers in solution but acquire helical conformation upon contact with phospholipid bilayers [5]. The interaction with bacterial cell 
membrane has been shown to be crucial for the activity of AMPs. Therefore, understanding the interaction of AMPs with the bacterial membrane and identifying the membrane properties/components responsible for the activity are of major importance to improve efficiency and decrease toxicity against the host.

\section{Interaction of AMPs with Cellular Membranes}

The plasma membrane is responsible for the compartmentalization of the cell and works as a selective barrier to control the gradients indispensable to life. It is mainly constituted by phospholipids and proteins, but each organism has its own characteristic membrane composition [6]. For example, eukaryotic membranes are mainly constituted by neutral glycerophospholipids, (such as phospholipids containing phosphatidylcholine or phosphatidylethanolamine head groups), whereas bacterial membranes possess a large percentage of anionic lipids such as cardiolipin and phospholipids containing phosphatidylglycerol $[5,6]$. Furthermore, bacteria have a more electronegative transmembrane potential than eukaryotic cells and possess negatively charged constituents on their surface (e.g., lipopolysaccharide and lipoteichoic acids in Gram-negative and Gram-positive bacteria, resp.) [5].

With a net positive charge, AMPs have selectivity towards the negatively charged surface of bacterial membranes over neutral eukaryotic membranes [5]. Nevertheless, AMPs may have distinct mode of actions and can be broadly divided into two main groups: AMPs that cause the disruption of the bacterial membrane or AMPs that cross the membrane and attack an intracellular target without disrupting the membrane [3].

The first interaction between peptides and membranes seems to be driven by electrostatic interactions between the phospholipids phosphate groups and the peptide hydrophilic aminoacids. After this first interaction, the peptide can insert in the membrane and either perturb the integrity of the plasma membrane or translocate into the cytoplasm of the cell in a nondisruptive way $[2,3,5]$.

The action of disruptive AMPs can be explained by the formation of pores in the membrane ("barrel stave" and "toroidal pore" mechanisms) or by accumulation of peptide molecules on the membrane surface ("carpet mechanism") $[3,5]$. On the other hand, nondisruptive peptides are able to cross the membrane and attack cytoplasmatic targets, such as nucleic acids and/or cellular proteins, or might have an inhibitory action on the synthesis of these compounds [5]. For instance, pyrrhocoricin, a prolinerich insect peptide, has been shown to bind the heat shock protein DnaK inhibiting chaperone-assisted protein folding [3]. The time necessary to detect loss of viability differs for disruptive and nondisruptive AMPs [3]. The disruptive peptides cause cell death in few minutes, whereas nondisruptive peptides act in a slower manner [3].

\section{Membrane Models}

As above referred, peptide-membrane interactions are important for the mechanism of action of AMPs. Biological membranes are complex and heterogeneous structures composed by hundreds of lipids and proteins [6,9] and with domains of distinct composition [9]. For that reason, simple model membranes are commonly used to distinguish the role of the different cell components on the mode of action of AMPs. The lipid charge, membrane viscosity, and the absence/presence of sterols are some properties that can be modulated [10]. In addition, model membranes are easily prepared with synthetic lipids or with lipids extracted from a certain type of cell [10]. 
The most commonly used membrane models are phospholipid vesicles that are classified based on their size and on the number of lamellae that constitutes them [11]. Unilamellar vesicles with a diameter equal or superior to $100 \mathrm{~nm}$ are referred to as large unilamellar vesicles (LUVs). Due to their nonconstrained curvature at molecular scale, LUVs have a large stability, possess identical lipid packing as biological membranes, and are preferred as a model [12]. These vesicles can be employed in the study of peptide-membrane interactions using biophysical techniques; in particular, the intrinsic fluorescence of peptides can be used to follow peptide-membranes interactions by means of fluorescence methodologies.

SUVs, small unilamellar vesicles, are also extensively used, but their small size $(20-50 \mathrm{~nm})$ and therefore their high surface curvature can lead to distorted lipid packaging and consequently to anomalous peptide packing and metastability [12]. Although SUVs are less prone to cause artifacts due to light scattering, the disadvantages mentioned above and the fact that the scattering artifacts associated with LUVs can be easily corrected make LUVs the preferred model [12]. It is also worth to mention that GUVs, giant unilamellar vesicles, with a diameter larger than $1 \mu \mathrm{m}$, can reach eukaryotic cells size and therefore are very useful for microscopy studies [11].

Human (e.g., erythrocytes) and bacterial cells can also be used to study peptide-membrane interactions and are important to validate the results obtained with simple model membranes. Nevertheless, the application of biophysical techniques with cells is limited; for instance, the intrinsic fluorescence of the peptide cannot be distinguished from the large cellular background fluorescence [13]. This limitation requires AMPs to be derivatized with extrinsic fluorophores such as rhodamine and nitrobenzoxadiazole; besides an increase in experimental and monetary requirements, the derivatization of peptides can alter their properties [13].

Theoretical models can also be used and in general are a good complement to experimental data. However, the small size of the virtual membrane patches, the limited number of peptide molecules, and the short-time scales that can be sampled restrict the extrapolation that can be made by these studies [14].

\section{Relevant Biophysical Studies for AMP-Membrane Interactions}

There are various biophysical techniques that can be employed to study peptide-membrane interactions. In this section some of these techniques will be briefly described.

\subsection{Fluorescence Spectroscopy}

The intrinsic fluorescence of peptides is exceptionally useful to study AMPs-membrane interactions as it provides structural and dynamic information in a noninvasive way [15]. Several fluorescence methodologies can be employed, and some will be addressed bellow.

\subsubsection{Partition Constant, $K_{p}$}

As referred above, AMPs have to interact with plasma membranes to exert their antimicrobial activity; therefore their extension of partition into the membranes is particularly relevant. Partition can be 
quantified by the determination of the partition constant $\left(K_{p}\right)$ defined as the ratio of the peptide molecules in the lipid $(L)$ over the peptide in the aqueous phase $(W)[10,16]$ :

$$
K_{p}=\frac{n_{S, L} / V_{L}}{n_{S, W} / V_{W}}
$$

where $n_{S, i}$ is the moles of solute present in the aqueous $(i=W)$ and lipid $(i=L)$ phases and $V_{i}$ is the water $(i=W)$ and $(i=L)$ lipid volume. When peptides partitionate into membranes, an increase in the fluorescence quantum yield and a spectral shift to more energetic wavelengths are usually observed (as exemplified with the AMP LEAP-2 in Figures 1(a) and 1(b). Therefore, the response is a combination of the signal emitted by free $\left(I_{W}\right)$ and bound $\left(I_{L}\right)$ peptides, and the equation (4.2) can be used to quantify the $K_{p}$ (Figure 1(a)) [16]:

$$
I=\frac{I_{W}+K_{p} \gamma_{L}[L] I_{L}}{1+K_{p} \gamma_{L}[L]}
$$

The peptide partition constant obtained for different lipid systems can be compared to evaluate lipid preference of the peptide. A greater affinity towards negatively charged membrane models (mimetic of bacteria) is expected for AMPs.

\subsubsection{Differential Quenching}

Quenching of the intrinsic fluorescence of peptides can be used to study their in-depth localization in the lipid membrane [17]. This method applies simple diffusional quenching concepts to the restricted dimensions of a bilayer [18]. The bilayer is treated as a slab in which fluorophores and quenchers exist with a determined in-depth distribution. The statistical distributions of the quenchers were previously determined by single-molecule Brownian dynamics simulations whereas the distribution of the fluorophores can be estimated using pairs of quenchers that are in general brominated or doxyl-derivatized acyl chains (in different positions of the acyl chain) in fatty acids or phospholipids [18]. One example is the pair 5 and 16-NS that are fatty acids derivatized with a doxyl group on the positions 5 and 16, respectively. 5-NS locates in the aqueous environment-membrane interface, whereas the 16-NS localizes in the hydrophobic core [17]. The relative degree of quenching between quenchers and fluorophores depends on the proximity between these molecules, and, therefore, fluorescence intensity will give information about the prevailing localization of the peptides in the membrane [17]. Additionally, a watersoluble quencher such as acrylamide can be used to determine if the fluorophore is accessible to the aqueous environment and, thus, complements this analysis.

\subsubsection{Membrane Permeabilization}

As previously mentioned, certain AMPs can cause the disruption of the plasma membrane by different proposed mechanisms. The ability of a specific peptide to induce the permeation/lysis of a certain model membrane can be quantified by the percentage of leakage induced by these peptides [19]. One frequently used method is to follow carboxyfluorescein fluorescence intensity [19]. This probe is 


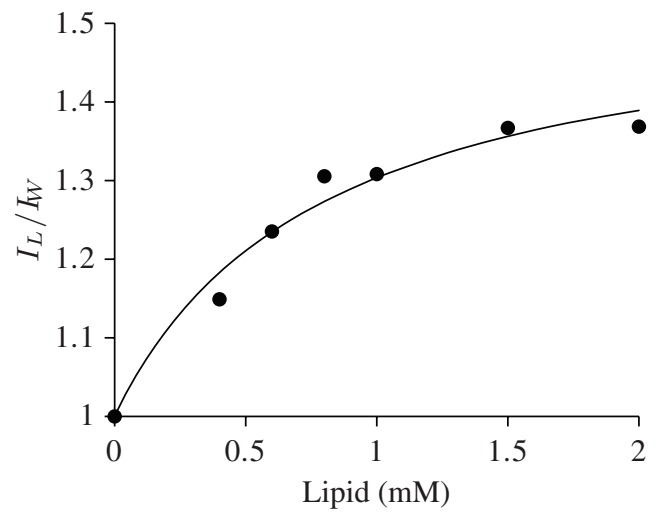

(a)

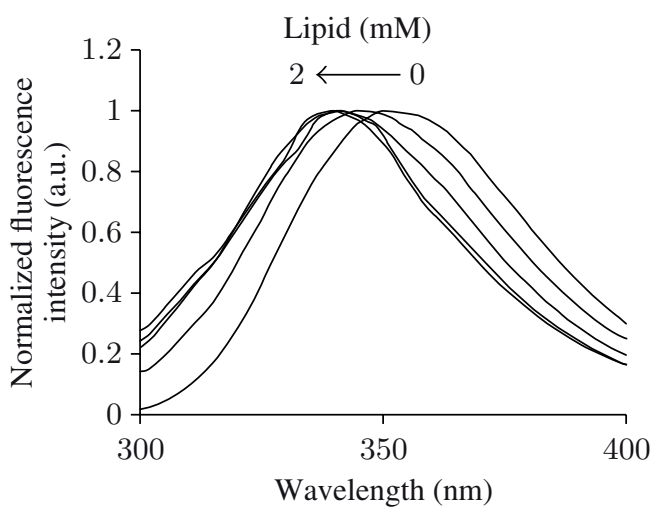

(b)

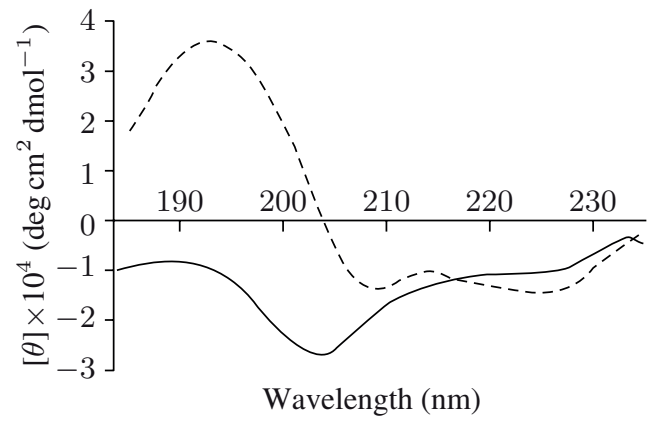

(c)

Figure 1: The use of spectroscopic methodologies to evaluate the interaction of AMPs with model membranes. (a) Partition of the AMP LEAP-2 into lipid vesicles followed by Trp fluorescence. An increase in the LEAP-2 fluorescence intensity upon titration with lipid vesicles is evident. The $K_{p}$ value can be determined applying (4.2). (b) Normalized fluorescence emission spectra of LEAP-2 illustrate a blue-shift upon titration with lipid vesicles. (c) Circular dichroism spectra of pardaxin 4 in the absence (full line) or presence of lipopolysaccharide (dashed line). Panels (a) and (b) were adapted from [7] and panel (c) was adapted from [8].

incorporated inside LUVs, being highly concentrated, and thus is self-quenching. However, when AMPs induce membrane leakage, carboxyfluorescein is released and diluted, which leads to an increase of fluorescence intensity proportional to the percentage of leakage [20]. Applying the equation (4.3), it is possible to calculate the leakage percentage induced by the peptide on a membrane with the composition studied:

$$
\% \text { Leakage }=\frac{\left(I-I_{0}\right)}{\left(I_{\text {positive control }}-I_{0}\right)}
$$

$I$ is the fluorescence intensity in the presence of the peptide, $I_{0}$ is the fluorescence intensity in the absence of peptide and $I_{\text {positive control }}$ is the fluorescence intensity in the presence of the positive control; usually Triton X-100 is used [20]. 


\subsubsection{Circular Dichroism}

Circular dichroism is a spectroscopic technique that can be used to estimate the overall secondary structure of peptides [21]. Peptide bonds are the most absorbing components in the far UV region (240-180 nm), and every secondary structure element has a characteristic spectrum [21]. The secondary structure of peptides can change upon insertion in lipid vesicles. Such alteration can be followed by circular dichroism and is relevant to understand the mechanism of action of AMPs. Figure 1(c) compares the secondary structure of pardaxin 4 in the absence and presence of a model of the outer membrane of Gram-negative bacteria [5]. A change from random coil to $\alpha$-helix structure upon interaction with the model membrane is evident.

Other studies, such as the orientation of peptides, are possible using spectroscopic techniques resorting to linear dichroism. They are out of the scope of this paper due to their specificity. The reader is referred to references $[22,23]$ for this subject.

\subsection{Atomic Force Microscopy}

Atomic force microscopy (AFM) can be used to obtain three-dimensional images of the microbial cell surface and of the lipid membrane. AFM images can be acquired in real time, under physiological conditions, with minimal sample preparation [24] and can be used to examine model membranes [25] or bacterial cells [2] treated with AMPs (as exemplified with the AMP BP100 in Figures 2(a) and 2(b)). Bacterial cell surface can exhibit changes in the shape and rugosity, whereas in model membranes the presence of holes and changes in the phase are possible effects. In addition, force measurements can be employed to study intra- and intermolecular forces [26] and measure mechanical properties of the bacterial cells after treatment with AMPs [24]. With this technique it is possible to directly compare the effects on model and cell membranes.

\subsection{Zeta Potential}

The superficial negative charge of bacterial membranes can be neutralized upon titration with cationic AMPs and this phenomenon can be followed by Zeta potential ( $\zeta$-potential) [27]. In solution, polyelectrolyte particles have a layer of ions at their surface that moves with the particles. Above this layer there are ions that do not move with the molecule, and electric potential existent in this boundary is referred to as $\zeta$-potential [27]. It has been previously observed that $\zeta$-potential of anionic lipid vesicles and bacterial cells becomes less negative upon increase of the peptide/lipid ratio. Such effect reflects the interaction between the peptide and the membrane as exemplified in Figure 2(c), with Escherichia coli cells titrated with the AMP BP100 [27]. The possibility of doing these studies with lipid vesicles and bacterial cells is a great advantage because it enables a direct comparison between the two systems. The $\zeta$-potential is measured using the Henry's relation:

$$
U_{E}=\frac{2 \varepsilon z f(\kappa \alpha)}{3 \eta}
$$




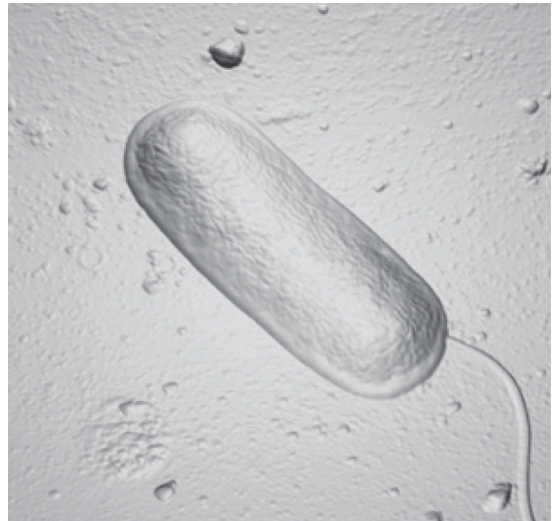

(a)

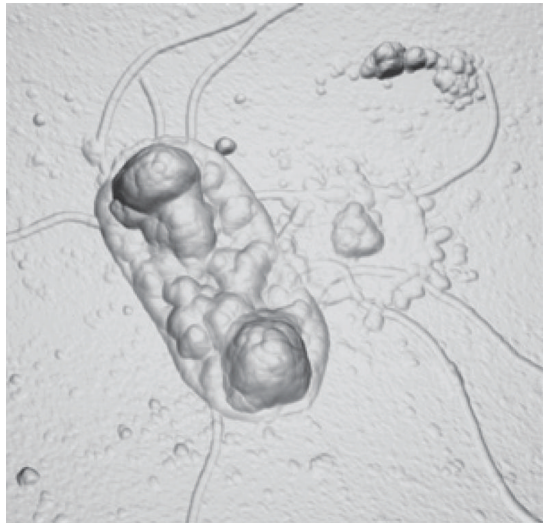

(b)

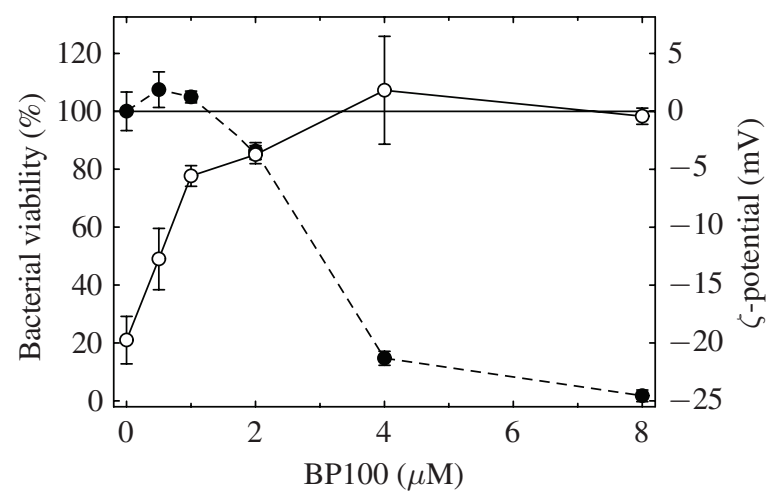

(c)

Figure 2: The use of bacterial cells in biophysical studies. Three-dimensional orthogonal projection of Escherichia coli cell (a) nontreated or (b) treated with the AMP BP100 obtained with AFM imaging. (c) Titration of Escherichia coli cells with BP100 induces an increase in the Zeta potential and a decrease in the bacterial viability, revealing that antibacterial activity correlates with the surface membrane charge neutralization. Panels (a)-(c) adapted from [2].

where $f(\kappa \alpha)$ is the Henry's function, $z$ is the $\zeta$-potential, $U_{E}$ is the electrophoretic mobility, $\varepsilon$ is the dielectric constant, and $\eta$ is the viscosity of the solution. $U_{E}$ is calculated by laser Doppler velocimetry in which the particle velocity is related to the frequency measured by variations of the intensity of the scattered light. For particles in aqueous solution the Henry's function takes the value 1.5, following the Smoluchowski approximation. When the particles are suspended in nonaqueous solution, the value of the function is 1, according to Huckel approximation [27].

\section{Conclusion}

With a large spectrum of activity, high efficiency and low toxicity, AMPs are a potential alternative to conventional therapies. Their mode of action is dependent on peptide-membrane interaction; therefore for a broad application of those peptides, to improve their activity and to reduce eventual toxicity, it 
is important to understand their mode of action. Biophysical techniques are useful tools to understand peptide-membrane interactions. Using lipid vesicles and fluorescence spectroscopy methodologies it is possible to obtain information on peptide-membrane affinity, peptide in-depth location, and membrane stability upon interaction of AMPs with model membranes. With circular dichroism spectroscopy the secondary structure motifs of the peptides can be identified, and alterations on the secondary structure upon contact with model membranes can be evaluated. On the other hand, $\zeta$-potential and AFM can be employed with either model membranes or biological cells. $\zeta$-potential can give details on superficial charge neutralization and with AFM, AMPs actions on model membranes and biological cells can be directly observed. Altogether, it is possible to conclude that biophysical techniques are valuable tools to study the mechanism of action of AMPs and other membrane-active peptides, as insights on the peptide-membrane interactions can be obtained.

\section{Acknowledgments}

S. Henriques is a Marie Curie International Outgoing Fellow within the 7th European Community Framework Program (PIOF-GA-2008-220318). Work in our laboratory on antimicrobial peptides is supported by a grant from the Fundação para a Ciência e Tecnologia, Portugal (PTDC/SAU-BEB/099142/ 2008).

\section{References}

[1] S. Thomas, S. Karnik, R. S. Barai, V. K. Jayaraman, and S. Idicula-Thomas, "CAMP: a useful resource for research on antimicrobial peptides," Nucleic Acids Research, vol. 38, supplement 1, pp. D774-D780, 2010.

[2] C. S. Alves, M. N. Melo, H. G. Franquelim et al., "Escherichia coli cell surface perturbation and disruption induced by antimicrobial peptides BP100 and pepR," Journal of Biological Chemistry, vol. 285, no. 36, pp. 27536-27544, 2010.

[3] J. P. Powers and R. E. Hancock, "The relationship between peptide structure and antibacterial activity," Peptides, vol. 24, no. 11, pp. 1681-1691, 2003.

[4] M. N. Melo, R. Ferre, and M. A. R. B. Castanho, "Antimicrobial peptides: linking partition, activity and high membrane-bound concentrations," Nature Reviews Microbiology, vol. 7, no. 3, pp. 245$250,2009$.

[5] M. R. Yeaman and N. Y. Yount, "Mechanisms of antimicrobial peptide action and resistance," Pharmacological Reviews, vol. 55, no. 1, pp. 27-55, 2003.

[6] G. van Meer, D. R. Voelker, and G. W. Feigenson, "Membrane lipids: where they are and how they behave," Nature Reviews Molecular Cell Biology, vol. 9, no. 2, pp. 112-124, 2008.

[7] S. T. Henriques, C. C. Tan, D. J. Craik, and R. J. Clark, "Structural and functional analysis of human liver-expressed antimicrobial peptide 2," ChemBioChem, vol. 11, no. 15, pp. 2148-2157, 2010.

[8] A. Bhunia, P. N. Domadia, J. Torres, K. J. Hallock, A. Ramamoorthy, and S. Bhattacharjya, "NMR structure of pardaxin, a pore-forming antimicrobial peptide, in lipopolysaccharide micelles: mechanism of outer membrane permeabilization," Journal of Biological Chemistry, vol. 285, no. 6, pp. 3883-3895, 2010.

[9] G. W. Feigenson, "Phase boundaries and biological membranes," Annual Review of Biophysics and Biomolecular Structure, vol. 36, pp. 63-77, 2007. 
[10] M. M. Ribeiro, M. N. Melo, I. D. Serrano, N. C. Santos, and M. A. R. B. Castanho, "Drug-lipid interaction evaluation: why a 19th century solution?" Trends in Pharmacological Sciences, vol. 31, no. 10, pp. 449-454, 2010.

[11] N. C. Santos and M. A. R. B. Castanho, "Liposomes: has the magic bullet hit the target?" Química Nova, vol. 25, pp. 1181-1185, 2002.

[12] A. S. Ladokhin, S. Jayasinghe, and S. H. White, "How to measure and analyze tryptophan fluorescence in membranes properly, and why bother?" Analytical Biochemistry, vol. 285, no. 2, pp. 235-245, 2000.

[13] S. T. Henriques, M. N. Melo, and M. A. R. B. Castanho, "How to address CPP and AMP translocation? Methods to detect and quantify peptide internalization in vitro and in vivo (review)," Molecular Membrane Biology, vol. 24, no. 3, pp. 173-184, 2007.

[14] H. Khandelia, J. H. Ipsen, and O. G. Mouritsen, "The impact of peptides on lipid membranes," Biochimica et Biophysica Acta, vol. 1778, no. 7-8, pp. 1528-1536, 2008.

[15] N. C. Santos and M. A. R. B. Castanho, "Fluorescence spectroscopy methodologies on the study of proteins and peptides. On the 150th anniversary of protein fluorescence," Trends in Applied Spectroscopy, vol. 4, pp. 113-125, 2002.

[16] N. C. Santos, M. Prieto, and M. A. R. B. Castanho, "Quantifying molecular partition into model systems of biomembranes: an emphasis on optical spectroscopic methods," Biochimica et Biophysica Acta, vol. 1612, no. 2, pp. 123-135, 2003.

[17] M. X. Fernandes, J. G. de la Torre, and M. A. R. B. Castanho, "Joint determination by Brownian dynamics and fluorescence quenching of the in-depth location profile of biomolecules in membranes," Analytical Biochemistry, vol. 307, no. 1, pp. 1-12, 2002.

[18] P. M. Matos, H. G. Franquelim, M. A. R. B. Castanho, and N. C. Santos, "Quantitative assessment of peptide-lipid interactions. Ubiquitous fluorescence methodologies," Biochimica et Biophysica Acta, vol. 1798, no. 11, pp. 1999-2012, 2010.

[19] E. E. Ambroggio, F. Separovic, J. H. Bowie, G. D. Fidelio, and L. A. Bagatolli, "Direct visualization of membrane leakage induced by the antibiotic peptides: maculatin, citropin, and aurein," Biophysical Journal, vol. 89, no. 3, pp. 1874-1881, 2005.

[20] M. M. Domingues, M. A. R. B. Castanho, and N. C. Santos, "rBPI 21 promotes lipopolysaccharide aggregation and exerts its antimicrobial effects by (hemi)fusion of PG-containing membranes," PLoS ONE, vol. 4, no. 12, Article ID e8385, 2009.

[21] S. M. Kelly and N. C. Price, "The use of circular dichroism in the investigation of protein structure and function," Current Protein and Peptide Science, vol. 1, no. 4, pp. 349-384, 2000.

[22] S. C. D. N. Lopes, E. Goormaghtigh, B. J. Cabral, and M. A. R. B. Castanho, "Filipin orientation revealed by linear dichroism. Implication for a model of action," Journal of the American Chemical Society, vol. 126, no. 17, pp. 5396-5402, 2004.

[23] S. C. D. N. Lopes and M. A. R. B. Castanho, "Overview of common spectroscopic methods to determine the orientation/alignment of membrane probes and drugs in lipidic bilayers," Current Organic Chemistry, vol. 9, no. 9, pp. 889-898, 2005.

[24] Y. F. Dufrene, "Atomic force microscopy, a powerful tool in microbiology," Journal of Bacteriology, vol. 184, no. 19, pp. 5205-5213, 2002.

[25] H. G. Franquelim, S. Chiantia, A. S. Veiga, N. C. Santos, P. Schwille, and M. A. R. B. Castanho, "Anti-HIV-1 antibodies 2F5 and 4E10 interact differently with lipids to bind their epitopes," AIDS, vol. 25, no. 4, pp. 419-428, 2011.

[26] N. C. Santos and M. A. R. B. Castanho, "An overview of the biophysical applications of atomic force microscopy," Biophysical Chemistry, vol. 107, no. 2, pp. 133-149, 2004.

[27] M. M. Domingues, P. S. Santiago, M. A. R. B. Castanho, and N. C. Santos, "What can light scattering spectroscopy do for membrane-active peptide studies?" Journal of Peptide Science, vol. 14, no. 4, pp. 394-400, 2008. 


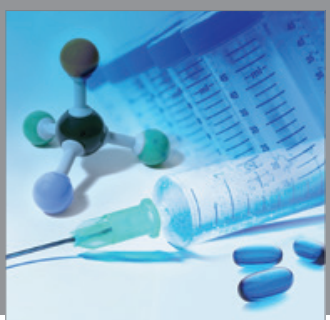

International Journal of

Medicinal Chemistry

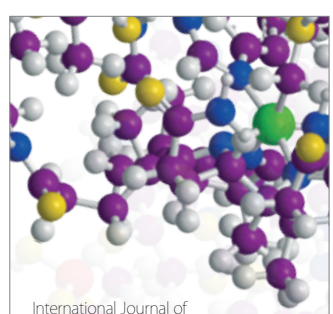

Carbohydrate Chemistry

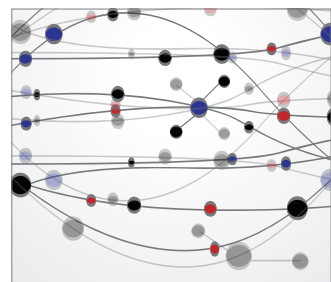

The Scientific World Journal
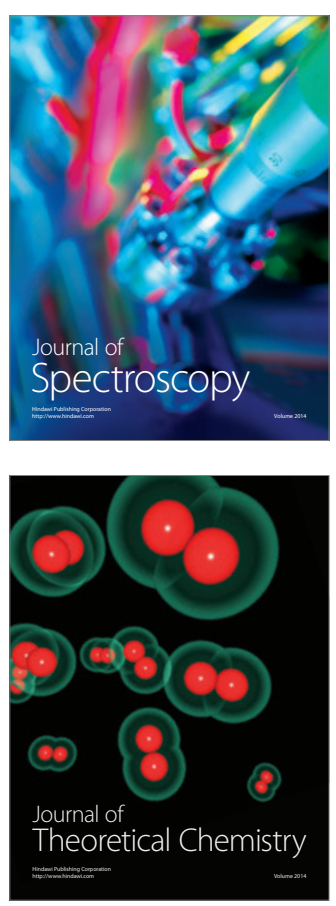
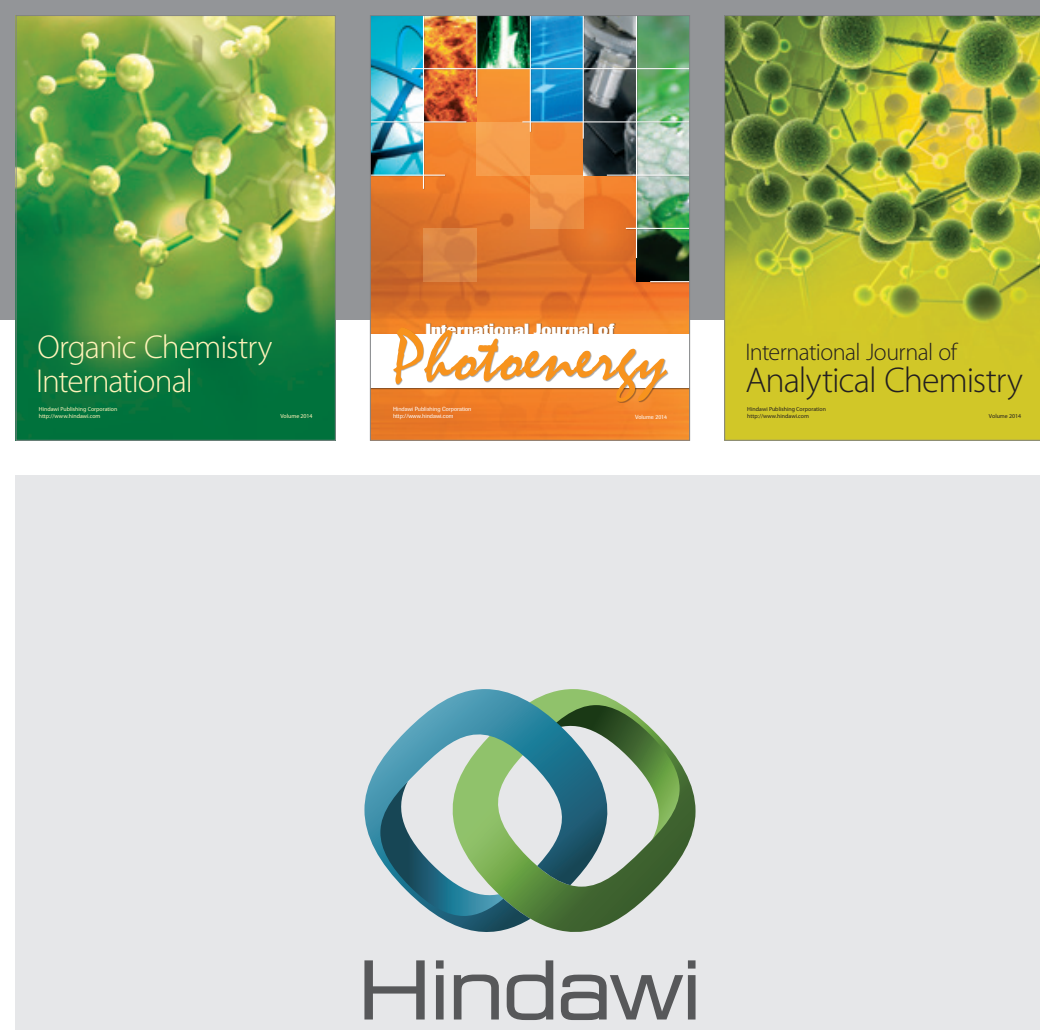

Submit your manuscripts at

http://www.hindawi.com
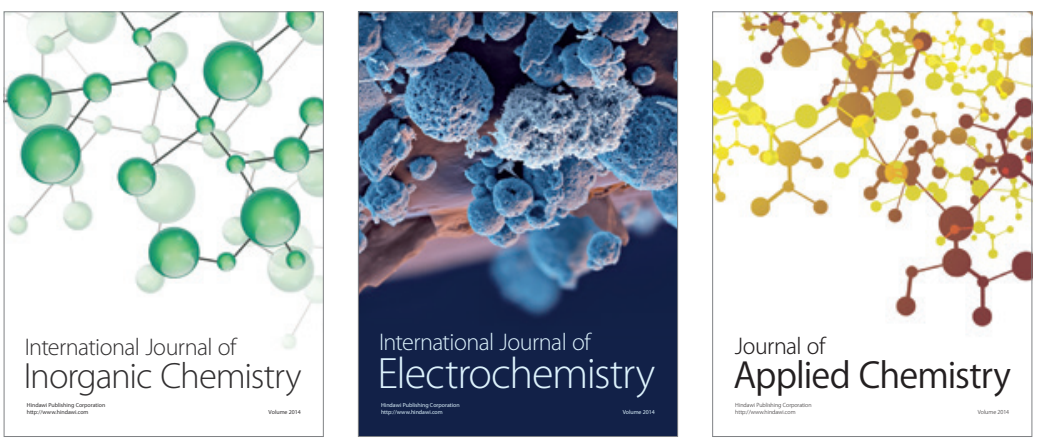

Journal of

Applied Chemistry
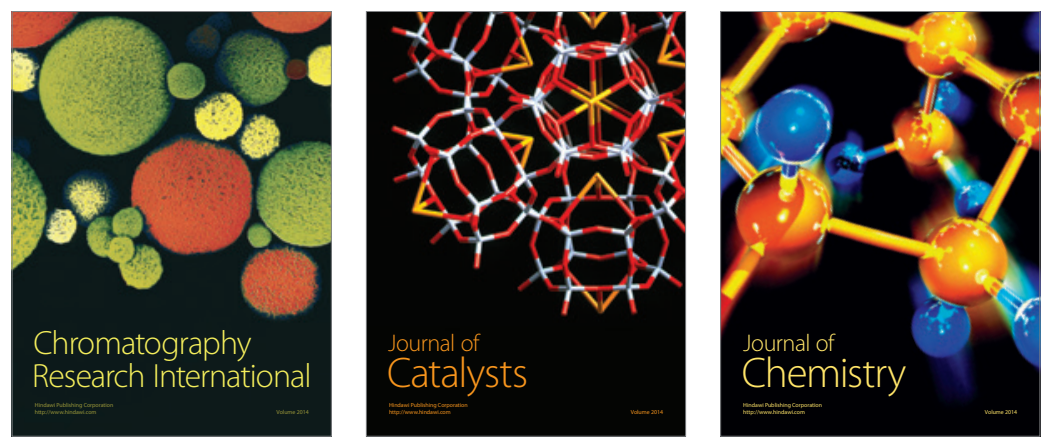
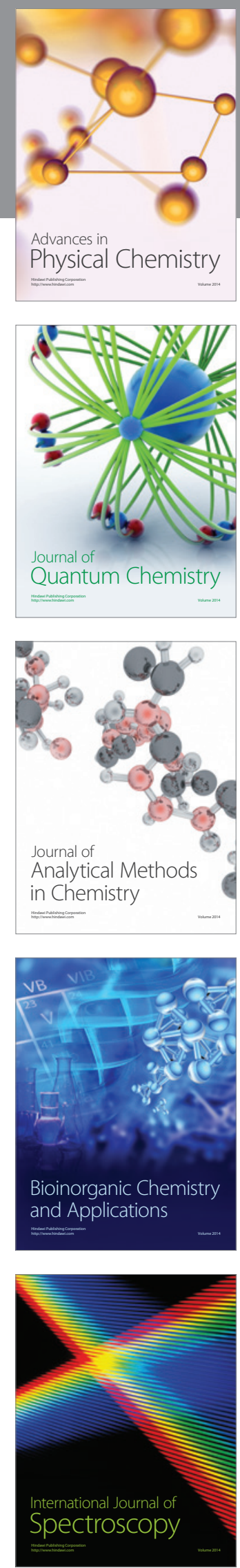